\title{
The calculation and analysis of the thermal efficiency equivalent heat drop for 600MW super-critical unit
}

\author{
Bing ZHANG \\ Liaoning Jianzhu Vocational University, Liaoyang 111000, China \\ 350993222@qq.com
}

\begin{abstract}
Keywords: $600 \mathrm{MW}$; the principle of thermal system;equivalent heat drop;thermal efficiency
Abstract: A comprehensive exposition on the calculation process of the principle equivalent heat drop thermodynamic system . Articles with equivalent heat drop method to calculate the thermal efficiency N600-24.2 / 566/566 type $600 \mathrm{MW}$ super-critical units, energy management group for this type of machine provides the basis for the data. At the same time gives a complete support system reasonably calculated to provide a reference for similar calculations.
\end{abstract}

\section{Introduction}

Equivalent heat drop method is the 70s of last century developed a thermal theory, is thermal systems analysis, a method of calculating the practicality and energy research. Equivalent heat drop method can be used to calculate the overall thermodynamic system can also be used for local quantitative calculation of the thermodynamic system ${ }^{[1]}$. Equivalent Heat Drop abandoned the disadvantages of the conventional calculation does not require recalculation of the overall system will be able to identify changes in the economy after the heat, with simple, convenient and accurate. In this paper, the new production in recent years, the main unit N600-24.2 / 566/566 type 600 MW super-critical unit with a thermal efficiency of equivalent heat drop method computer group, and provides a basis for the same type of thermal economic management unit. In particular, the calculation process is very typical secondary treatment system, computer aided systems equivalent heat drop method for providing a reference.

\section{Equivalent heat drop theory}

Equivalent heat drop method is based on the principles of thermodynamics thermal power conversion, taking into account the characteristics of the quality of equipment, thermal system structure and parameters, a rigorous theoretical deduction, several thermal analysis parameters derived for the degree of conversion and utilization of thermal energy a method. It can be used to calculate the overall thermodynamic system can also be used for quantitative analysis of local thermodynamic system. It is essentially a heat balance method of energy conversion. Its advantage is simple local operations instead of the entire complex computing systems, research and systematic change only those parts locally relevant quantitative.

For pure condensing steam turbine, a new steam power made $1 \mathrm{~kg}$ equals its enthalpy drop [1], namely:

$$
h=h_{m s}-h_{c}
$$

Formula: h of steam for the new work done; the beginning of a new steam enthalpy into the turbine; for turbine exhaust enthalpy, heat recovery for power extraction turbine $1 \mathrm{~kg}$ of new steam 
done for

$$
h=\left(h_{m s}-h_{c}\right)-\alpha_{1}\left(h_{1}-h_{c}\right)-\cdots \cdots-\alpha_{i}\left(h_{i}-h_{c}\right)
$$

Formula: $\mathrm{h}$ is the work done by the new steam; for a new beginning enthalpy of steam entering the turbine; for turbine exhaust enthalpy; i-th stage turbine extraction steam enthalpy; as Extraction share.

Comparison of the formula (1) and (2) : the work done by extraction turbine heat recovery steam less than $1 \mathrm{~kg}$ of pure new condensing steam turbine, for its enthalpy of pure condensing steam turbine drop differ, the enthalpy drop of formula (2) is called the equivalent enthalpy drop. Equivalent enthalpy drop of meaning: the power extraction turbine heat recovery steam $1 \mathrm{~kg}$ new made equivalent $(\mathrm{kg})$ steam enthalpy drop new direct Condenser.

\section{MW unit equivalent heat drop overall computing}

The main parameters of the unit ${ }^{[2,3]}$

Turbine Type:600-24.2/566/566

Steam initial parameter: $p_{0}=24.2 \mathrm{MPa}, t_{0}=566^{\circ} \mathrm{C}$

Reheat steam parameters: pressure segment cold, cold zone temperature ${ }^{\circ} \mathrm{C}$, hot section pressure, hot leg temperature ${ }^{\circ} \mathrm{C}$, the exhaust pressure.

Extraction and seal parameters in Table 2-1 and Table 2-2.Mechanical efficiency, efficiency of the generator were taken for $\eta_{m}=0.99 、 \eta_{g}=0.988$

Table 2-1 heat recovery steam extraction parameters for each segment

\begin{tabular}{|c|c|c|c|c|c|c|c|c|c|}
\hline Item & Unit & \multicolumn{8}{|c|}{ Heat recovery steam extraction parameters for each segment } \\
\hline Heater No. & - & H1 & $\mathrm{H} 2$ & $\mathrm{H} 3$ & $\mathrm{H} 4$ & H5 & H6 & $\mathrm{H} 7$ & $\mathrm{H} 8$ \\
\hline $\begin{array}{l}\text { Extraction } \\
\text { pressure }\end{array}$ & $\mathrm{MPa}$ & 5.62 & 4.33 & 2.31 & 1.16 & 0.438 & 0.128 & 0.0619 & 0.0237 \\
\hline $\begin{array}{l}\text { Extraction } \\
\text { temperature }\end{array}$ & ${ }^{\circ} \mathrm{C}$ & 349.2 & 314.9 & 483.9 & 379.6 & 261.3 & 139.8 & 86.8 & 63.8 \\
\hline \multicolumn{10}{|c|}{ Table 2-2 Parameters seal } \\
\hline Item & & Unit & & $\alpha_{s g 1}$ & \multicolumn{3}{|c|}{$\alpha_{s g 2}$} & \multicolumn{2}{|c|}{$\alpha_{s g 3}$} \\
\hline Source & & & \multicolumn{2}{|c|}{$\begin{array}{l}\text { High pressure steam } \\
\text { leakage pole }\end{array}$} & \multicolumn{3}{|c|}{$\begin{array}{c}\text { After the } \\
\text { low-pressure cylinder } \\
\text { shaft seal leakage }\end{array}$} & \multicolumn{2}{|c|}{$\begin{array}{l}\text { Steam leakage } \\
\text { between the high } \\
\text { pressure cylinder }\end{array}$} \\
\hline Seal of steam & & & \multicolumn{2}{|c|}{0.0006339} & \multicolumn{3}{|c|}{0.001038} & \multicolumn{2}{|c|}{0.00007958} \\
\hline Seal steam enthalp & & $\mathrm{kJ} / \mathrm{kg}$ & \multicolumn{2}{|c|}{3396.0} & \multicolumn{3}{|c|}{2753.7} & \multicolumn{2}{|c|}{2993.7} \\
\hline To place & & & \multicolumn{2}{|c|}{$\mathrm{H} 8$} & \multicolumn{3}{|c|}{ SG } & \multicolumn{2}{|c|}{$\mathrm{H} 2$} \\
\hline
\end{tabular}




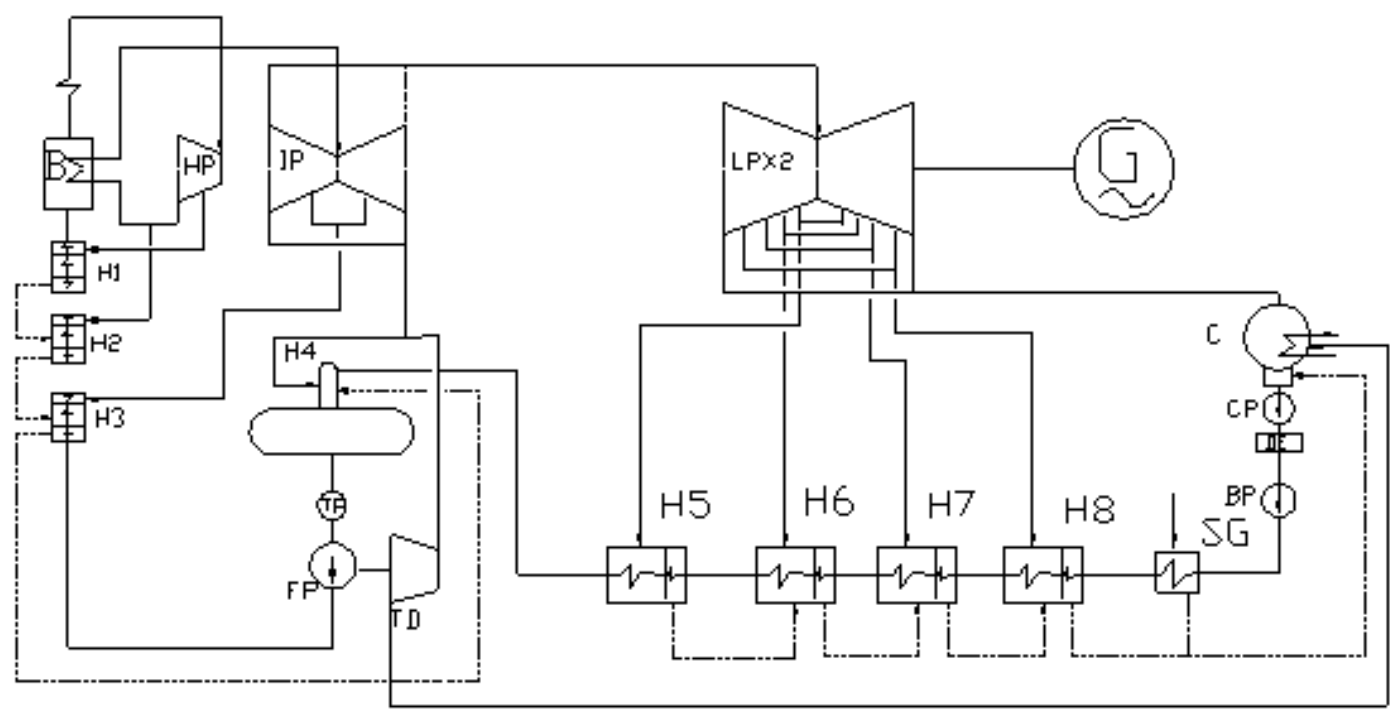

Fig. 1 600MW thermal system schematic diagram of principl

(1)According to the known parameters $\mathrm{p} 、 \mathrm{t}$ 在 $\mathrm{h}$-s diagram drawn on a steam turbine expansion line, get a new steam enthalpy, enthalpy levels Extraction and exhaust enthalpy, and reheat steam specific enthalpy rise. Also according to $\mathrm{p}, \mathrm{t}$ check the steam table to obtain the above enthalpy.

$h_{0}=3396.0 \mathrm{~kJ} / \mathrm{kg}, \quad h_{\mathrm{rh}}^{\text {in }}=2993.7 \mathrm{~kJ} / \mathrm{kg}, \quad h_{r h}^{\text {out }}=3596.0 \mathrm{~kJ} / \mathrm{kg}, h_{j}$ and $h_{c}$ see the value shown in section 2.3. $q_{r h}=3596.0-2993.7=602.3 \mathrm{~kJ} / \mathrm{kg}$.

(2)Calculate point parameters export water heaters and related hydrophobic enthalpy enthalpy steam table or investigation, according to the unit back to the heating system are listed in Table 2-3.

Table 2-3 regenerator system calculates the point parameters

\begin{tabular}{|c|c|c|c|c|c|c|c|c|}
\hline Item & Unit & $\mathrm{H} 4$ & H5 & H6 & $\mathrm{H} 7$ & $\mathrm{H} 8$ & SG & $\mathrm{C}$ \\
\hline Extraction pressure & $\mathrm{MPa}$ & 1.16 & 0.438 & 0.128 & 0.0619 & 0.0237 & / & 0.0049 \\
\hline Extraction Pressure & $\%$ & 5 & 5 & 5 & 5 & 5 & / & / \\
\hline $\begin{array}{l}\text { Side pressure steam } \\
\text { heater }\end{array}$ & $\mathrm{MPa}$ & 1.10 & 0.417 & 0.122 & 0.0588 & 0.0225 & 0.098 & / \\
\hline Extraction enthalpy & $\mathrm{kJ} / \mathrm{kg}$ & 3218.7 & 2986.5 & 2753.7 & 2632.6 & 2498.6 & / & 2325.8 \\
\hline Seal steam enthalpy & $\mathrm{kJ} / \mathrm{kg}$ & / & / & / & / & / & 3396.0 & / \\
\hline Saturation temperature & ${ }^{\circ} \mathrm{C}$ & 184.0 & 145.1 & 105.2 & 85.4 & 62.6 & 99 & 32.5 \\
\hline Saturated water enthalpy & $\mathrm{kJ} / \mathrm{kg}$ & 780.89 & 611.12 & 441.06 & 357.63 & 262.03 & / & 136.20 \\
\hline Heater difference & ${ }^{\circ} \mathrm{C}$ & 0 & 2.8 & 2.8 & 2.8 & 2.8 & / & 0 \\
\hline Heater outlet temperature & ${ }^{\circ} \mathrm{C}$ & 184.0 & 142.3 & 102.4 & 82.6 & 59.8 & 33.3 & / \\
\hline $\begin{array}{l}\text { Water heater side } \\
\text { pressure }\end{array}$ & $\mathrm{MPa}$ & 1.10 & 1.8 & 1.8 & 1.8 & 1.8 & 1.8 & / \\
\hline $\begin{array}{c}\text { Water heater outlet } \\
\text { enthalpy }\end{array}$ & $\mathrm{kJ} / \mathrm{kg}$ & 780.8 & 598.9 & 429.4 & 346.0 & 250.3 & / & 136.2 \\
\hline Drain cooler end poor $\vartheta$ & ${ }^{\circ} \mathrm{C}$ & l & 5.6 & 5.6 & 5.6 & 5.6 & / & / \\
\hline $\begin{array}{l}\text { Drain cooler outlet water } \\
\text { temperature }\end{array}$ & ${ }^{\circ} \mathrm{C}$ & / & 108.0 & 88.2 & 65.4 & 38.9 & / & / \\
\hline $\begin{array}{l}\text { Hydrophobic drain } \\
\text { cooler enthalpy }\end{array}$ & $\mathrm{kJ} / \mathrm{kg}$ & / & 453.0 & 369.5 & 273.8 & 162.7 & / & / \\
\hline
\end{tabular}




\section{Simple calculation and data processing}

The parameters of the original parameters and thermal equilibrium law, upon finishing simple calculation results are shown in Table 2-4, the auxiliary steam parameters as shown in Table 2-5.

Table 2-4 regenerative system parameters and the main parameters of finishing

\begin{tabular}{ccccccc}
\hline Heater No. & $\begin{array}{c}\text { Extraction } \\
\text { enthalpy } \\
\mathrm{kJ} / \mathrm{kg}\end{array}$ & $\begin{array}{c}\text { Hydrophobic } \\
\text { enthalpy } i_{r} \\
\mathrm{~kJ} / \mathrm{kg}\end{array}$ & $\begin{array}{c}\text { Export water } \\
\text { enthalpy } t_{r} \\
\mathrm{~kJ} / \mathrm{kg}\end{array}$ & $\begin{array}{c}\text { Steam heat } \\
\text { release } \\
q_{r}=h_{r}-i_{r} \\
\mathrm{~kJ} / \mathrm{kg}\end{array}$ & $\begin{array}{c}\text { Hydrophobic } \\
\text { heat } \\
\text { release } \\
\gamma_{j}=i_{r-1}-i_{r} \\
\mathrm{~kJ} / \mathrm{kg}\end{array}$ & $\begin{array}{c}\text { Feedwater } \\
\text { enthalpy } \\
\text { rise }\end{array}$ \\
$\begin{array}{cccccc}\tau_{r}=t_{r}-t_{r+1} \\
\mathrm{~kJ} / \mathrm{kg}\end{array}$ \\
\hline 4 & 3218.7 & 598.9 & 813.7 & 2619.8 & - & 181.9 \\
5 & 2986.5 & 453.0 & 598.9 & 2533.5 & - & 169.5 \\
6 & 2753.7 & 369.5 & 429.4 & 2384.2 & 83.5 & 83.4 \\
7 & 2632.6 & 273.8 & 346.0 & 2359.0 & 95.7 & 95.7 \\
8 & 2498.6 & 162.7 & 250.3 & 2335.9 & 111.1 & 111.0 \\
\hline Exhaust & 2325.8 & - & 136.2 & - & - & - \\
\hline
\end{tabular}

Table 2-5 Auxiliary Steam System

\begin{tabular}{ccccc}
\hline Name & sign & Share\% & Enthalpy & Whereabouts \\
\hline A high bar steam leakage & $\alpha_{m g A}$ & 0.0006 & 3396.0 & Low plus 8 \\
$\begin{array}{c}\text { B high pressure steam } \\
\text { leakage between } \\
\text { cylinders }\end{array}$ & $\alpha_{z f B}$ & 0.00008 & 2993.7 & High plus 2 \\
$\begin{array}{c}\text { After the low-pressure } \\
\text { cylinder seal C }\end{array}$ & $\alpha_{z f C}$ & 0.001 & 2753.7 & Seal Heater \\
\hline
\end{tabular}

Extraction heater heat release, the water heater and the hydrophobic heat release enthalpy rise in heater. Table 2-4.

\section{calculated}

Extraction Extraction equivalent enthalpy drop and efficiency of each segment is

(1)eighth pumping and pumping efficiency equivalent enthalpy drop

$$
\begin{aligned}
& H_{8}^{0}=h_{8}-h_{c}=2498.6-2325.8=172.8 \mathrm{~kJ} / \mathrm{kg} \\
& \eta_{8}^{0}=\frac{H_{8}^{0}}{q_{8}}=0.073976
\end{aligned}
$$

(2)Seventh paragraph pumping and pumping efficiency equivalent enthalpy drop

$$
\begin{aligned}
& H_{7}^{0}=h_{7}-h_{8}+H_{8}^{0}-\gamma_{8} \eta_{8}^{0} \\
& \eta_{7}^{0}=\frac{H_{7}^{0}}{q_{7}}=\frac{298.58094}{2359.0}=0.126571
\end{aligned}
$$

(3)Sixth paragraph pumping and pumping efficiency equivalent enthalpy drop

$$
\begin{aligned}
& H_{6}^{0}=h_{6}-h_{7}+H_{7}^{0}-\gamma_{7} \eta_{7}^{0} \\
& \eta_{6}^{0}=\frac{H_{6}^{0}}{q_{6}}=\frac{407.5684}{2384.2}=0.1709456
\end{aligned}
$$

(4)Fifth paragraph of the pumping and pumping efficiency equivalent enthalpy drop

$$
H_{5}^{0}=h_{5}-h_{6}+H_{6}^{0}-\gamma_{6} \eta_{6}^{0}
$$


$\eta_{5}^{0}=\frac{H_{5}^{0}}{q_{5}}=\frac{626.0945}{2533.5}=0.247126$

(5)Fourth paragraph pumping and pumping efficiency equivalent enthalpy drop

$$
\begin{aligned}
& H_{4}^{0}=h_{4}-h_{c}-\sum_{r=5}^{8}\left(\tau_{r} \eta_{r}^{0}\right) \\
& \eta_{4}^{0}=\frac{H_{4}^{0}}{q_{4}}=\frac{816.4311}{2619.8}=0.311639
\end{aligned}
$$

\section{Reheat steam heat absorption incremental computing}

Cold reheat steam reheaters paragraph above exclusion endothermic steam extraction caused $1 \mathrm{~kg}$ increments calculated as follows:

$\Delta Q_{z r-2}=\sigma=q_{r h}=602.3(\mathrm{~kJ} / \mathrm{kg})$

\section{New steam equivalent enthalpy drop calculation}

(1) New steam gross equivalent heat drop

$$
\begin{aligned}
H_{M} & =h_{0}+\sigma-h_{c}-\sum_{r=4}^{8}\left(\tau_{r} \eta_{r}^{0}\right) \\
& =1539.3440(\mathrm{~kJ} / \mathrm{kg})
\end{aligned}
$$

(2) Various additional components acting losses caused

A leak in the high bar acting loss

$$
\begin{aligned}
\pi_{1} & =\alpha_{m g A}\left[\left(h_{m g A}+q_{r h}-h_{c}\right)-\left(h_{m g A}-h_{2}\right) \eta_{8}^{0}-\left(h_{8}-h_{c}\right)\right] \\
& =0.9086(\mathrm{~kJ} / \mathrm{kg})
\end{aligned}
$$

After the low-pressure cylinder seal leakage acting steam loss of C

$$
\begin{aligned}
\pi_{2} & =\alpha_{z f C}\left[\left(h_{z f C}-h_{c}\right)-\left(h_{z f C}-h_{8}\right) \eta_{8}^{0}-\left(h_{8}-h_{c}\right)\right] \\
& =0.2452(\mathrm{~kJ} / \mathrm{kg})
\end{aligned}
$$

Feedwater pump turbine steam consumption losses:

$$
\begin{aligned}
& \tau_{b}=813.7-780.8=32.9(\mathrm{kj} / \mathrm{kg}) \\
& \pi_{b}=\tau_{b}\left(1-\eta_{3}^{0}\right)=\tau_{b}=32.9(\mathrm{kj} / \mathrm{kg})
\end{aligned}
$$

\section{Turbine efficiency calculation installed}

New steam net equivalent heat drop $\mathrm{H}$ :

$$
\begin{aligned}
H & =H_{M}-\sum \pi_{i} \\
& =1539.3440-(32.9+0.9086+0.2452)=1505.2902(\mathrm{~kJ} / \mathrm{kg})
\end{aligned}
$$

Circulation Heat:

$$
\begin{aligned}
Q & =h_{0}+\alpha_{r h} \sigma-h_{f w} \\
& =3175.37698(\mathrm{~kJ} / \mathrm{kg})
\end{aligned}
$$

Turbine absolute internal efficiency:

$\eta_{i}=\frac{H}{Q}=\frac{1505.2902}{3175.37698}=47.4051 \%$ 
Absolute Power Turbine Efficiency:

$\eta_{e}=\eta_{i} \eta_{m} \eta_{g}=0.474051 \times 0.99 \times 0.988=46.3679 \%$

Turbine heat rate;

$q=\frac{3600}{\eta_{e}}=\frac{3600}{0.463679}=7764.0[\mathrm{~kJ} /(\mathrm{kW} \cdot \mathrm{h})]$

Turbine steam consumption rate:

$d=\frac{q}{q_{0}}=\frac{7764.0}{3175.37698}=2.4451[\mathrm{~kg} /(\mathrm{kW} \cdot \mathrm{h})]$

\section{Conclusions}

Equivalent heat drop is a simple method to calculate the thermal efficiency, in line with the real thermodynamic system. In this paper, the equivalent heat drop method gives the current main unit $600 \mathrm{MW}$ supercritical unit heat consumption for basic data management and thermal efficiency. The successful application of equivalent heat drop method based on the method requires a deep understanding, especially critical computing systems is the relationship between the secondary use of the method to success. In this paper, the calculation of the common auxiliary systems, and for similar calculations provide a reference.

\section{References}

[1] Lin Wan Chao. Thermal power plant system energy theory [M]. Xi'an: Xi'an Jiaotong University Press .1994.

[2] Zheng Ti Kuan. Thermal power plants [M]. Beijing: China Electric Power Press, 2001

[3] Li Qing, Gong Wei Ping. Power plants and energy-management technology index [M]. Beijing: China Electric Power Press, 2006. 